plenty of room for the heads of the metatarsal bones. The soles must also be thick. As an adjunct a rubber bandage or a leather band may be placed firmly around the bases of che metatarsal bones, so as to separate the heads as much as possible. If the pain is not relieved by the above treatment excision of the head of one of the metatarsal bones is necessary, and I should remove that one which had the largest corn over it.

Weymouth-street, $\mathrm{W}$,

\section{NOTES OF A CASE OF PREMATURE LABOUR PRESENTING SOME UNUSUAL FEATURES. ${ }^{1}$}

BY JOHN MOCAW, M.D. R.U.I.,

PHYSICIAN TO THE BELFAST HOSPITAL FOR SICK CEILDREN.

THE interest in the following case lies in the fact that a septic condition had already become established before the advent of labour, if, indeed, it was not itself the cause of the premature delivery.

A married woman aged thirty-six years sought advice on April 26th, 1894, regarding the state of her health, which had not been satisfactory of late, and gave the following history. She had had a family of eight and was pregnant of her ninth child. All had been born at full term and were quite healthy. Since she became pregnant she had not felt well, so that her usual household duties had become a burden to her. She had lost flesh and her natural healthy complexion had given place to one of bilious or coppery tint. From about one month after menstruation ceased she became aware of a new sensation on the right side of the abdomen, which later increased to well-marked and constant pain from the third month of pregnancy until I first saw her on the above date. She was unable to assign any cause for this pain, and she did not remember having sustained a blow or fall, though I questioned her very closely on this point. She came to me late in the evening because she had noticed some water running from her, and this had continued since the morning. I visited her on the following morning, when her temperature was $101.5^{\circ} \mathrm{F}$. and the pulse 90. The tongue was furred and the bowels were constipated. The fotal heart sounds were weak; there was slight general abdominal tenderness, with one very tender spot on the right side about the middle of a line running from the right anterior superior spine to the umbilicus. Examination of the liver and spleen gave negative results and the chest organs were healthy; her urine was scanty and highly coloured, acid in reaction, and free from albumin and sugar. Examination per vaginam revealed that the os was slightly dilated, but not sufficiently so to admit the finger. With a speculum I was able to satisfy myself that the water she spoke of as coming from her was flowing from the uterine cavity; this fluid was rose-coloured, quite watery, and had a peculiar heavy, sweet, sickly odour. She was ordered a dose of castor oil and to be kept warm in bed. At 4 P.M. the same day I was sent for and $I$ found her suffering severely from pains in her back, arms, and legs ; her temperature had risen to $102.5^{\circ}$ and her pulse to 110 . She bad much frontal headache and a dry, hot skin, but the backache put ererything else into the shade and she was crying out urgently for something to relieve it. I ordered her to have ten grains of sodium salicylate with ten minims of liquor opii sedativus. every two hours till relieved. I found that the os had dilated so as easily to admit the finger, and a head presentation was diagnosed. I was called to see the patient again at 10 P.M., when I found her in labour with regular and good pains, and after this the following points were noted:-1. She became more and more restless and complained bitterly of the severity of the pains, although they did not appear to me to be advancing the progress of the head in a proportionate degree. 2. She began to have shiverings which increased in severity until 1130 , when she had a well-marked rigor, her temperature at this time being $105.8^{\circ}$ in the axilla. 3. At 11.45 she was delivered of a living male child who had reached about the middle of the seventh month of gestation. The child was healthy-looking

1 A paper read before the Ulster Medical Society on May 3rd, 1895. in every respect and free from skin eruption of any kind. 4 Immediately on expulsion of the fotus there was a gush of fluid corresponding in appearance with the fluid I have before described as flowing from the uterus the same morning, but with the difference that it was now intensely fœtid. On proceeding to express the placenta I found it to be adherent and requiring the introduction of the hand. The greater part of it was readily removed and was quite healthy, but a portion of it about the size of a hen's egg I had considerable difficulty in picking off the uterus, and when removed it was of the same colour as the fluid and its odour more foetid, if that were possible. While removing this portion of placenta she complained that I was giving her great pain, and said, "That's where the pain has been all the time." I washed out the uterus with very hot water and gave ber a dose of ergot. On the following morning her temperature had fallen to $102.5^{\circ}$; she was very weak and as the discharge from the uterus was foetid I washed it out with hot perchloride of mercury solution ( 1 in 2000). On the morning of the 29th I was summoned hurriedly as "she had fainted," and on arrical I found her recovering from a severe attack of syncope. No cause was assigned for the seizure and examination failed to detect anything pointing in the direction of embolism. She made progress, but two days afterwards was seized with another attack of syncope, this time, however, brought on by her turning herself in bed. Again she recovered under the free use of stimulants. From this time forward she made steady progress and was allowed to sit up in bed with the help of a bed-rest on the fourteenth day. On the morning of May 16th, however, I was sent for with the same message as beforenamely, that "she had fainted"-and on my arrival she was dead. On inquiry I was told that she had passed a good night and had only a short time previously partaken of her breakfast with relish; her sister, who was in the room underneath her bedroom, heard a sound as of someone falling on the floor overhead and running up at once she found the patient lying on the floor at the bedside; she raised the alarm and everything was done that had proved successful on the two previous occasions, but this time without avail and the patient never spoke. She had evidently risen to urinate (as the chamber was by her bedside) and died from syncope. This brings me to the end of this rather novel case, in my experience, and I would be greatly indebted to anyone who can throw any light on what seems to me to have been a preseptic condition. Fordyce Barker has seen some cases of a similar nature, but he does not explain how the fotid condition of a portion of the placenta in my case came about. Can any of tie readers of THE LANCE'T do so?

Belfast.

\section{NOTES ON RINGWORM OF THE SCALP.}

BY W. T. FREEMAN, F.R.C.S. ENG.

IF much has been written upon the subject of this parasitic disease of the scalp there is at all events excuse for saying more, for it is admittealy still a very tronblesome disease to cure. Nevertheless, I venture to believe that it should not be the bugbear that it is to so many of us. Personally I no longer dread it, and, indeed, am only too pleased to receive as many cases for treatment as I can get. There is not any royal road to the cure. Each case has to be treated on its own merits, but there are certain general points that invariably require attention. Firstly, although it is a purely local disease, yet there are general conditions that assist in supplying a suitable soil for the growth of the parasite. Thus anything that fosters damp surroundings is undoubtedly a favourable element for fungoid development. Damp houses, ill-ventilated cellars, a damp soil, are all conditions that affect the epithelium of the scalp as well as that of the body generally. We want an absolutely dry, not a moist skin, to assist our attack. If possible, then, the patient should be placed in a honse the internal sanitary arrangements of which are beyond suspicion; its situation should be fairly high and admitting of plenty of wind access; and the foundations should be let into gravel or chalk. I believe that an inland residence is superior to a seaside one, and that the presence of salt in a moist seaside atmosphere, which has been asserted to be helpful in the care of 
tinea of the scalp, is a fallacy. To salt freely and strongly applied $I$ have given an extensive trial. I have found it the least useful of any treatment I have adopted. Indeed, I believe it to be useless and shall never waste any more time with it.

Are there any" other general conditions that should be attended to? Well, the most difficult child to cure is of the fair-haired scrofulous type ; therefore I believe that cod-liver oil for ordinary anti-scrofulous treatment may be aidful. I am in the habit of occasionally giving small doses of Fowler's solution three or four times a day to delicate anæmic children unless there are concomitant skin conditions forbidding its use. I do not consider this empirical dosing, for arsenic is not only an excellent tonic and blood restorer, but its effects upon the skin generally are well known. One other general point. I always order my patients upon all posisible occasions to have the scalp freely exposed to the influence of the sun and air; and this for two reasons, the first being that nothing saprophytic can enjoy light and air, and the second that the less freely the scalp is covered the less moisture will there be in the intercellular spaces. This question of a hot moist scalp or of a cool dry one is of immense importance, and yet we still hear of instructions implying a more or less impermeable head covering. Of course, from time to time the head must be washed in order to assist in the removal of effete material from the hair and skin, but the subsequent drying is at least of equal importance to the cleansing, and the instructions as to this must be most carefully given. Also, there are times when a cap must be worn. It should be a light one, and should be relined daily with thin lint or permeable linen, or, if expense bas to be considered, with paper.

As to all the many remedies that have been vaunted as cures for ringworm most of them will cure it in a more or less reasonable space of time. But it is not as a rule the individual remedy in itself that relieves; its method of application, together with the preparation of the part for its attack, really deserve the credit. I could not point to any one application and say this has done more good for me than any other. All the following remedies will cure it in from six weeks to three months, and occasionally a little more time than tbree months may be occupied. Most of them require expert knowledge both as to when they are to be applied and when to be left off. Phenol, pure, or employed as a lotion or as an ointment. The lotion may be anything from half strength to 1 in 20 and the ointment from twenty grains to two drachms to the ounce. Lotions should not be applied more than twice daily, and the whole scalp must be well dried twenty minutes after application. Ointments should be rutbed in in the interval several times in the daywell rubbed in. All this the patient's attendants can do. Another useful lotion is 1 in 1000 perchloride of mercury, which is to be used after the same fashion as the carbolic lotion. Various ointments can be used in the place of the carbolic ointment, such as ten grains and upwards of salicylic acid, twenty grains to one and a half drachms of sulphur, or ammoniated mercury ten grains to one drachm to the ounce of vaseline or of vaseline and lanoline. Creasote, ichthyol, nitrate of mercury, chrysarobin, the oleates of mercury, each in various combinations. When to use one and when the other does not generally very much matter, but sulphur or salicylic acid when the combination of the disease is seborrhcic and ammoniated mercury when it is inclined to be pustular. Probably a mixture of lanoline and vaseline is as pene rating a base as we can get. The medical attendant should once or twice a week, at his discretion, make a stronger application to the worst spots; such are absolute phenol, a salicylic paste, Coster's paste, and croton oil. I do not consider that croton oil stamps out an early case any quicker than does any other strong remedy. All these powerful remedies require careful watching as to results. Thin-skinned children and patients under four or fire years of age require very careful handling, jet such treatment is essentially necessary. The irritation or inflammation produced has to be carried just far enough to destroy the fungus and yet be confined within the limits of safety. A chrysophanic ointment is another strong and good apolication-the strength should be from twenty grains to two drachms to the ounce; it may quickly produce an excess of inflammation. Given then a case seen for the first time some such directions as these should be given:- The whole head is to be scrubbed daily with soft soap, carbolic soap, or ccal-tar soap, preceded every few days with a rubbing in of turpentine till the child calls out. The application given to the patient is to be rubbed in several times daily. The head is to be kept shaved and the whole scalp kept very dry at all times; ironing is a helpful drying process. The lining of caps is to be renewed daily. Combs and brushes are to be rigorously disinfected or destroyed; they will not, of course, be required for use during the first few weeks of treatment. The patient is to be seen by the medical man himself at least once a week. The medical man will prepare the way by epilation and scarification, with suitable instruments. When the disease is apparently cured a fortnight should be allowed with only a daily soap washing and the hair should be allowed to grow. At the end of the week it should be inspected and again at the end of the fortnight. If then nothing wrong can be discovered the patient must be similarly inspected at the end of another week and fortmight. If at the end of the month no diseased hair is discoverable the patient may be certified as cured. Very frequently, however, at the end of apparent cure and after the lapse of from a week to a month a diseased hair or a few diseased hairs may again peep up, being pushed forward from the follicular depths. What is to be done? Well, if there are not more than a dozen or so hairs they must be individually destroyed and the patient watched for a further three weeks. Electrolysis has been recommended for their destruction. Personally, I apply a thin, red-hot wire to the mouth of the follicle and push the wire home. The parts should be anæsthetised previously with carbolic acid or cocaine, but I have done it without a local anæsthetic. In some such cases I have successfully simply dug out, as it weye, the orifice of the follicle and applied phenol or salicylic paste by means of a thin probe. If after all treatment has been stopped I can find at the end of a fortnight no diseased bairs, or only an odd one, I am content to allow the patient to mix with others. I attack any unsound hair and also insist upon seeing the patient weekly or thereabouts within the following three weeks. These odd diseased hairs require most careful looking after. Sir Dyce Duckworth's chloroform test assists in their discovery. If in doubt, too, there is the microscope, but bairs that have been subjected to treatment are very diffeult material for the demonstration of the fungus.

A neglected case of ringworm never seems to lose its infective power, but a well-treated case nearly cured-that is to say, cured to the extent of only an odd diseased broken hair being discoverable-is harmless, provided that the broken hair or hairs be destroyed and the head generally be treated nnce a day with a disinfectant wash. Nevertheless, I do not allow a child to return to scbool until three weeks after the last broken bair was discovered.

I have lately had a troublesome case under my care, but I permitted the patient to go home at the end of three months. His home bejng some three hours? journey from my house $I$ alloweä him to come at the end of a fortnight to see me, and again at the end of a fut ther two weelss; upon the first occasion I extracted two bairs and destroy ed the follicular remains. This ended the diseased condition and the patient is now back at schoo'. It was a very bad disseminated case, which had been under treatment for a year before his medical attendant kindly sent him to me. It takes from two to four months to cure these disseminated cases. This I hold to be an excellent result, but one that should be obtained. The treatment deperds ucon the personal attention of the surgeon and upon the assiduous, patient efforts of the attendants. For attendants I employ trained nurses. In al but the early cases, which should be cured in from a month to six weeks, a more or less inflammatory method must be employed. Just how far this may go, when it must be stopped, and the strength of the application suited to the particular case, - all these tbings require an experienced hand, who has to be bold without being rash, and per. severing, yet knowing when he bas done enongh.

I have written and thought here only of private cases. Out-patient cases of ringworm of the scalp are still very troublesome to cure. The reasons are obviovs. Their business is not to take trouble for themselves or even to allow it to be taken for them. I would once more empbasise the fact that the successful treatment of ringworm depends upon the personal efforts of the surgeon and unon his untiring endeavours to get his instructions carried our.

At the present time, with the assistance of $\mathrm{Mr}$. K. W. Goadby of Guy's Hospital, I am making tube cultivations of 
the fungus: The results are stained and examined with $a_{1} \frac{1}{1}$ in. oil immersion. Later we hope to publish some notes. Reading.

\section{LEPPOSY TREATED BY THYROID GLAND.}

Br C. B. MAITLAND, M.R.C.S. ENG., L.R.C.P. EDIN., SURGEON-MAJOR, I.M.S.

CASE 1.-A Mussulman aged eighteen years, an only child, was brought to me on May 5th, 1894, for treatment. The family history was of no interest. He said that he was born at Nimach, still lived in the house he was born in, had never been more than a few miles away, ate the same food as other people of his class, and knew no lepers. Five years ago he first felt a sensation of heat and formication all over the limbs and body and noticed that he perspired much more than he used to do. Next he found that his hands and feet were becoming rather numb and that he did not feel things so distinctly as formerly. A year ago, without any previous staining, his superciliary ridges became thick and lumpy and the hair fell off. Fis voice at this time became weak and husky, without pain or cough. He complained chiefly of a "creepycrawly" feeling all over when he was in the suu, continuous perspiration, and a heavy feeling in his extremities. was healthy in every way with the exception of the symptoms due to leprosy. His eyebrow prominences were luwpy and hairless, his nostrils were wide open, the skin of his face was ratber lumpy, dusky, and sbining, and the lobes of his ears and his back were slightly nodular. The mucous membrane of his nose was pale and atrophied and that of his fauces pale and codematous. The skin of his fingers and toes and that between the nails and over the last joints was smooth and shiny and the last phalanges generally were wather bulbous. He was not impotent. Gentle pressure probably the ulnar-caused pain. He began with half a raw sheep's thyroid gland on May 5 th weigbing about a drachm. On the 11th be cummenced to take a whole two-drachm gland daily. On the 13th he said that for the last three days he bad not felt the "crawly" feeling and had sweated less. On the $23 r d$ he stated that the numbness of his hands was passing away. On the 28 th his eyebrow prominences were disbe looked, and said he felt, much better. Considerable pressure on the above-mentioned nerve did not cause pain. The tubercles on the other parts and the neuritic look of his digits were the same as before. On the 31st there was a winus on the under surface of the last phalanx of the third toe of the right foot leading down to the bone. He said that four or five days previously be noticed a painless pimple there that burst the same day. Probing gave a litule pain. On June 4th a typical leprous ulcer and sinus appeared on the left great toe. His voice seemed to be getting huskizr. On the 11th both the above sinuses bad healed without loss of bone. The tubercles on the face were disappearing On July 10th Surgeon-Captain Whitcombe, I.M.S, who had been carrging on the treatment since the last note was made, reported that the voice huskiness had nearly gone. There were no ulceration and no tubercles and the ulnar nerve was not nearly so tender on pressure. On Aug. 10th the patient returned to hospical after three weeks' absence. His voice was huskier and th $;$ ulnar nerve more sensitive. There was no retrogression otherwise. The treatment was re-commenced. On Sept. $15 \mathrm{~h}$ the report said: "Tubercles none. Skin supple and stiny appearance of phalanges almost entirely disappeared. Voice greatly improved. A good deal of pressure can be borne on ulnar nerve without pain. Face is still somewhat livid-looking. The patient expresses himself as feeling in much better health than when he began treatment."

CASE 2.-The patient was a Hindu aged thirty-fire years, the youngest of six children. He was born, and had always lived, in the house he was now in, and had never been more than thirty miles from Nimach. His uncle died a leper in this same house thirty years ago The patient's two brothers and three sisters were all brought up with him and all had healthy families; one of the brothers died from ferer and one from an accident. The patient had been married eighteen years and was childless but not impotent. His wife was healthy. His father died from fever fourteen years ago; his motber was alive and well. He had never associated with any leper except the above-mentioned uncle. About four years previously dark stains appeared on his face, neck, and back. A year later tubercles rose on these stains and he felt crawling sensations in the limbs. The tubercle grew larger and fresh ones came on the ears, chin, and nose About six months ago his voice became husky. He was a striking case of elephantiasis leontina. In addition his back was covered with tubercles and his fingers and toes presented the same appearance as those of the other patient; the skin over the deltoid muscles was lichenous. He complained chiefly of aching pains and numbness in the limbs, perspirations, and formication. Treatment began on May $25 \mathrm{th}$ with one raw sheep's thyroid gland daily. On the 31 st he said the perspirations, formication, and feeling of internal heat were less. The conjunctivæ were very much paler. On June 3rd he was ordered to take two glands daily. On the 11th the patient said he could now move the skin of his face easily, that it felt lighter, and that the tubercles were smaller. I could not see this myself, but the skin did look healthier. On July 15th Surgeon-Captain Whitcombe, who had been carrying on the treatment since the last note, reported that the skin of the face was much healthier, less livid and nodular. There were no formication or sweats. The patient stated that the pains in the limbs were very slight now, that he was not so easily fatigued, and that there was more feeling in his fingers. On Sept. 15th it was reported that the skin was looking very healthy and the tubercles were distinctly less prominent. The conjunctiva were very slightly injected. The voice was much less husky than when the treatment was commenced; sensation was much improved and the pains in the limbs had almost gone.

Remarks. - The thyroid gland certainly has had a beneficial influence on these two men. Even putting aside the patients' own statements as dictated by mere politeness there was the obvious improvement in the skin as evidenced by the effect on the tubercles and the ulceration. The ulcers certainly appeared on the Mrassulman's toes when be was fully under the influence of the glacd, but it is allowable to suppose that they bad commenced deeply some time previously. There were no symptoms of tbyroidism, although the Hindu was taking two glands daily from June 3rd to Sept. 15th. The glands were pounded up raw with sugar and water. These cases are very incomplete, but they are interesting as showing that thyroitd gland certainly has some effect on leprosy even when given to patients who continue their usual home life while undergoing treatment. I wish to convey my best thanks to Surgeon-Captain Whitcombe.

In continuation of the above I regret that circumstances have prevented my following up the above cases. I saw them in the spring of 1896 and found them apparently well and able to earn their living. As far as I could judge by inspection the disease had not advanced and the men said they had been better ever since the treatment and wished to begin again. If I get an opportasity I will make further trials with thyroid gland and with mercurg.

Suakin.

Glasgom Elstern Medical Societr.-At a meeting of this society, held on Wednesday, Oot. 21st, the following gentlemen were elected office-bearers for the ensuing year:- Retired President, Dr. William Patrick. President, Dr. Aslexander Patterson. Vice-President, Dr. William Findlay. Secretary, Dr. R.M'C. Service. Treasurer, Dr. William Buchanan. Reporting Secretary, Dr. J. Wilson Mathie. Members of Council, Dr. MIunro, Dr. Dunlop, Mr. W. L. Muir, Dr. William Muir, Dr. J. B. Stewart, and Dr. Black.

Brigaton Fospital Saturuat Fund-As a result of thi ; year's Hospital Siturday collection at Brighton a sum of $£ 716$ has been apportioned arnong fourteen medical charities of the town. Last year the sum of £1030 was available for this purpcse. This year $£ 278$ go to the Sussex County Hospital and $£ 115$ respectively to the Brighton, Hove, and Preston Dispen:ary arid the Children's Hospital. Last year the figure: were $£ 400$ and $£ 165$ respectively. Fospital Sunjay has not yet arrited. 